\title{
Recovery from Acute Malocclusion in Temporomandibular Disorders with Stabilization Splint: Case Report
}

\author{
Ji-Rak Kim \\ Department of Dentistry and Oral Medicine, School of Medicine, Daegu Catholic University, Daegu, Korea
}

Received March 1, 2021

Revised March 12, 2021

Accepted March 15, 2021

Correspondence to:

Ji Rak Kim

Department of Dentistry and Oral Medicine,

School of Medicine, Daegu Catholic

University, 33 Duryugongwon-ro 17-gil,

Nam-gu, Daegu 42472, Korea

Tel: +82-53-650-4285

Fax: +82-53-622-7067

E-mail: essay2ndtree@daum.net

https://orcid.org/0000-0002-1326-3948
Various conditions such as pain or effusion of temporomandibular joint, degenerative condylar resorption, and articular disc displacement can be a cause of malocclusion. However, the reasons of occlusal changes are ambiguous in some patients. Unexpected occlusal change in patients with or without temporomandibular disorder (TMD) symptom was mostly caused by masticatory muscular disorders. This article reports two cases of recovery of occlusal relationship in TMDs patients after stabilization splint therapy. Stabilization splint therapy could be useful in certain conditions of occlusal changes in TMD.

Key Words: Dental occlusion; Masticatory muscles; Occlusal splint; Temporomandibular joint disorders

\section{INTRODUCTION}

Temporomandibular disorder (TMD) is characterized by dysfunction of the temporomandibular joint (TMJ) and adjacent masticatory muscles. The etiology and pathophysiology of TMD are not yet fully understood, but various biological, environmental, and psychosocial aspects are involved. The Symptoms of TMD are such as pain, joint sound, restricted mandibular movement, and occlusal disturbances.

Many TMD patients complain of their uncomfortable occlusion or sudden occlusal change. Pain or effusion of TMJ may influence mandibular position, thus possibly leading to bite change [1]. Degenerative changes of mandibular condyle can also lead to changes in occlusal relationships [2]. These occlusal imperfections are easily elucidated by a consequence of TMD. However, the reasons of occlusal changes are ambiguous in some patients.

Masticatory muscles also have the important function of equilibrium in the occlusion. It is natural that occlusion can be altered in patients with myofascial pain of masticatory muscle [3]. On the contrary, we can see the patients have occlusal change without pain. Most reversible changes can be recovered for a certain period of time without any kind of treatment. If the occlusion never comes back, then the clinician needs to do an appropriate treatment. Here, we present the two cases of recovery from acute malocclusion in TMDs with stabilization splint.

\section{CASE REPORT}

\section{Case 1}

A 34-year-old woman visited the department of oral medicine at the Daegu Catholic University Hospital with a complaint of occlusal change. She explained that the change started around three months ago, and she didn't have any pain or other symptoms of TMD. Clinical, radiographic, and magnetic resonance imaging examinations were performed. Her jaw shifted very slightly to the right side and all the left molar did not contact while the right 
teeth contacted evenly (Fig. 1). There were no significant finding in the radiographs (Fig. 2). The MR images showed partial anterior disc displacement of the right condyle. We didn't find other signs of TMD except for the occlusion. A study cast was made to analyze her occlusion. The contact was sound in the study cast so she did not need selective tooth grinding or prosthetic treatment.

She was prescribed with non-steroidal anti-inflammatory drug (NSAIDs, aceclofenac $200 \mathrm{mg}$ \#2/day), muscle relaxant (clonazepam $0.5 \mathrm{mg}$ \#1/days). She was instructed to perform a daily moist hot pack. After 2 weeks of the treatment, she did not respond. We started stabilization splint therapy. After 3 months of splint wearing, the occlusion returned to normal temporarily but the condition failed to maintain. At that time, we could recognize that she has bruxism from the facet on the splint surface (Fig. 3). To reduce the activity of the masticatory muscle, botulinum toxin (Botox; Allergan, Inc., Irvine, CA, USA) was injected to both temporalis and masseter every 25 units.

She suffered from right lower molar $(\# 46,47)$ pain due to secondary caries during the splint treatment and we performed root canal therapy. Because of unstable occlusion, we fabricated a temporary crown and waited for recovery of occlusion. After 5 months of splint therapy and 2 months of botulinum toxin injection, the occlusion got back and kept in its natural position. Then we finished the crown prosthesis (Fig. 4). She is wearing the stabilization splint to protect the damage from bruxism.

\section{Case 2}

A 66-year-old man visited the clinic with pain on the left TMJ and occlusal change. He had intermittent pain that had begun 2 years ago. He had undergone physical therapy

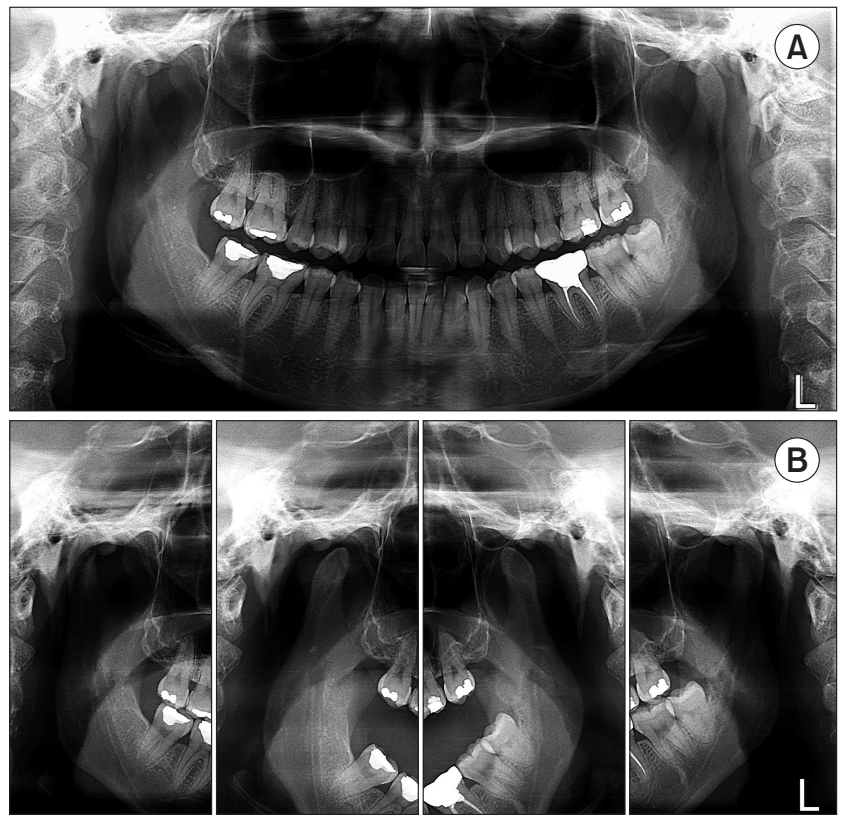

Fig. 2. Case 1. Panorama (A) and temporomandibular joint panorama (B) image taken at the first visit.
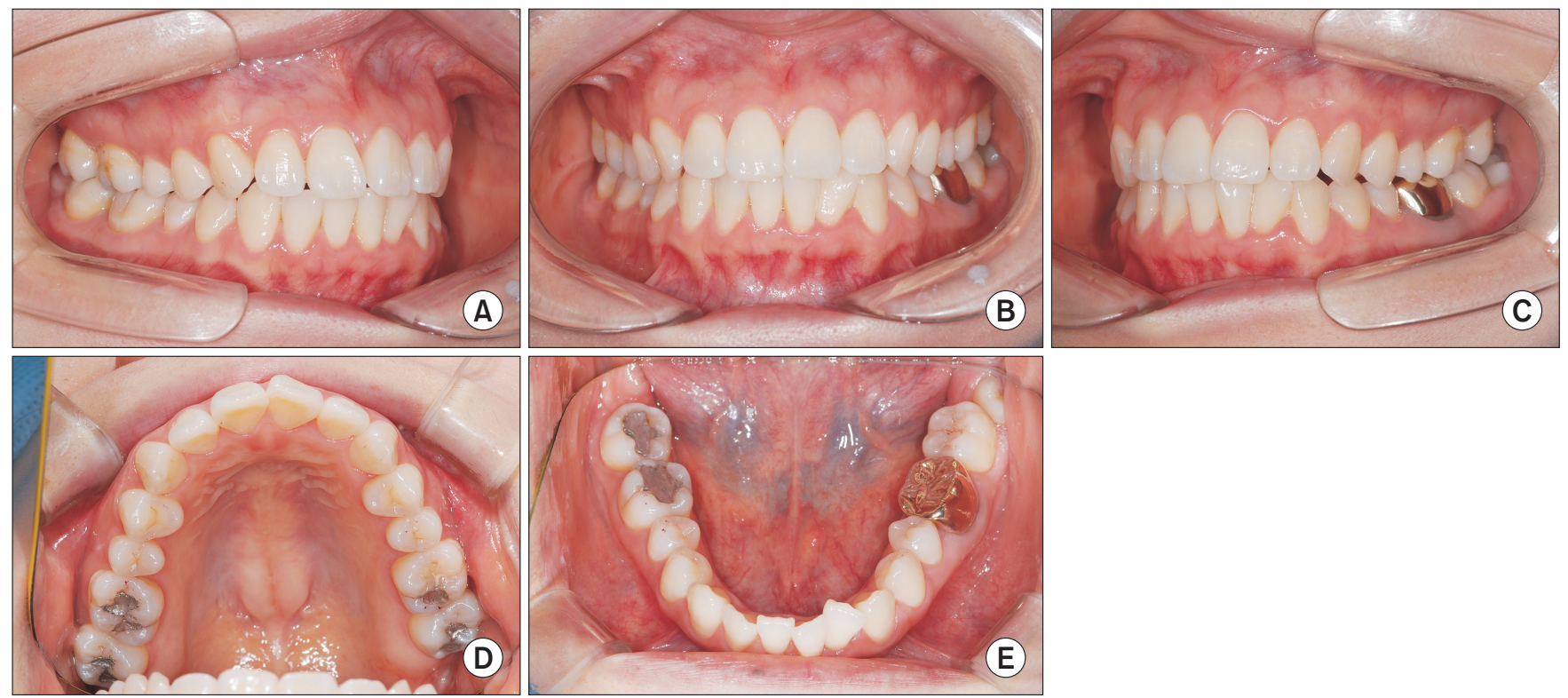

Fig. 1. Case 1. Clinical photographs of the right (A), frontal (B), left (C), upper (D), and lower (E) taken at the first visit. 

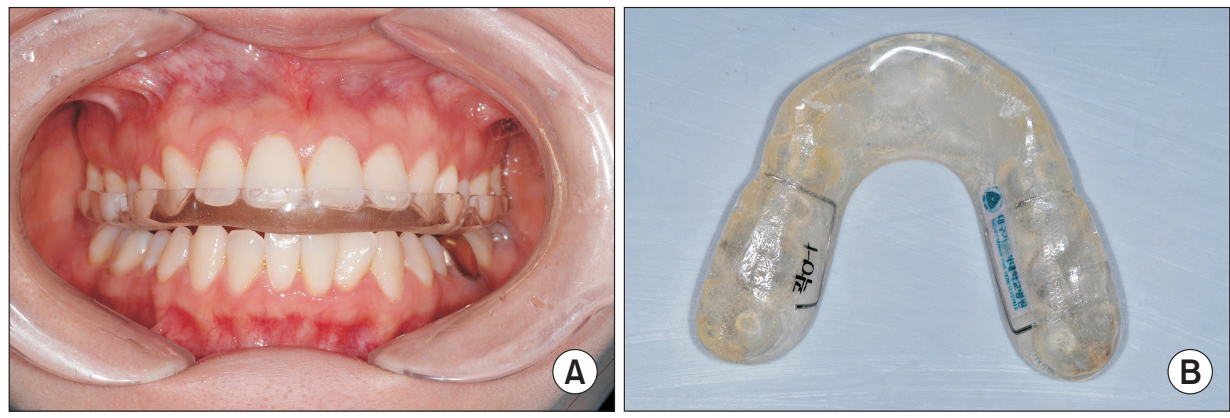

Fig. 3. Stabilization splint (A) and Bruxofacet (B).
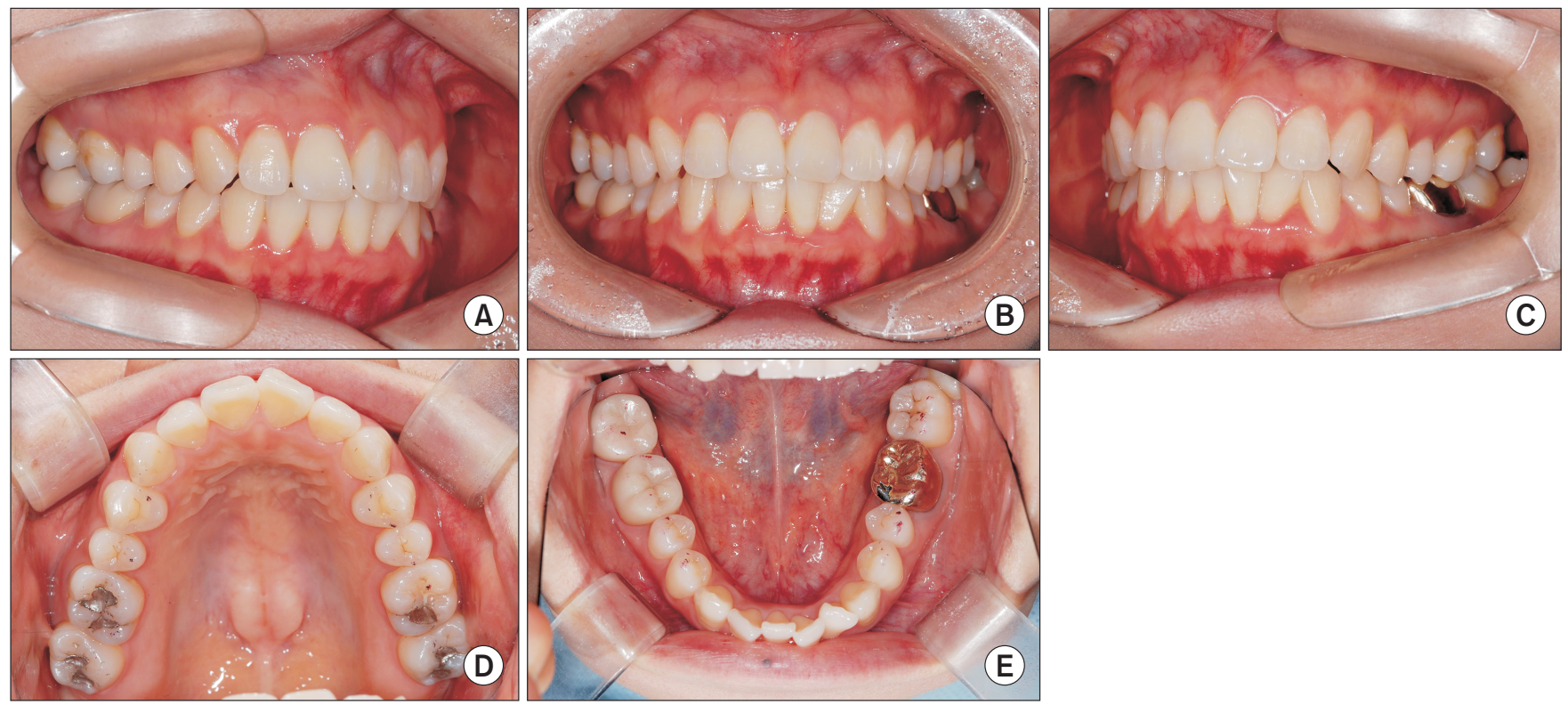

Fig. 4. Case 1. Clinical photographs of the right (A), frontal (B), left (C), upper (D), and lower (E) taken after splint treatment.
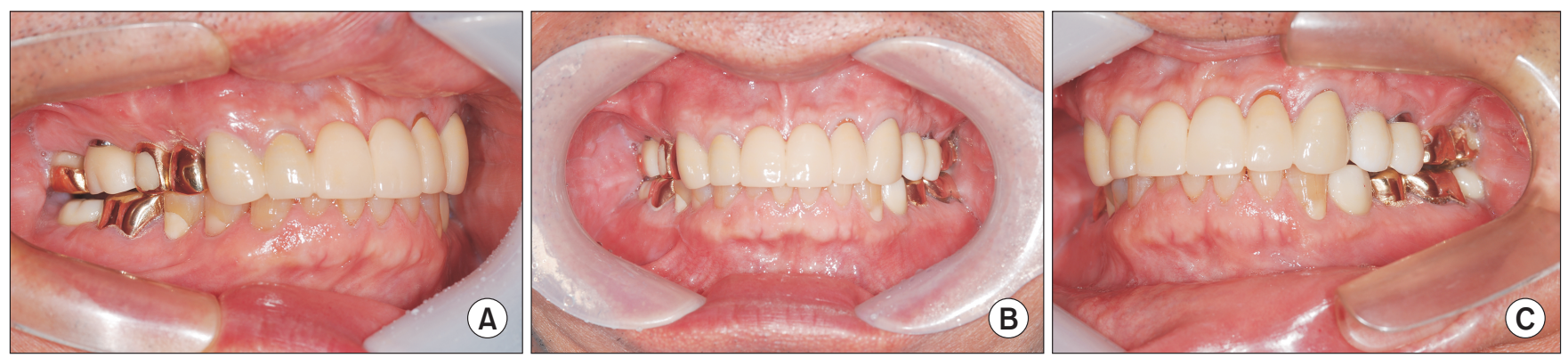

Fig. 5. Case 2. Clinical photographs of the right (A), frontal (B), and left (C) taken at the first visit.

and medication at a dental clinic, and pain on left TMJ had subsided. He had been using a stabilization splint for TMJ health maintenance without a regular checkup. He noticed that occlusal change several months ago and he stopped wearing the splint. Clinical and radiographic examinations were performed. TMJ examination showed pain on his left TMJ. His jaw shifted to the anterior side and all the molar teeth did not contact (Fig. 5). There were no significant finding in the radiographs (Fig. 6). The splint he used had a poor fit on his dentition and did not contact with opposite teeth evenly. The occlusion was sound in the diagnostic cast.

We instructed him to stop using the ill-fitting splint and self-management exercise including the reduction of TMJ 
use and control of parafunctional habits. He was prescribed NSAIDs (aceclofenac $200 \mathrm{mg}$ \#2/day). His pain on Lt TMJ decreased after NSAIDs medication, but occlusion did not recover. We started stabilization splint therapy. After 2 months of splint wearing, the posterior open bite was solved (Fig. 7). All the premolar and molar were occluding in natural position. His pain and malocclusion had been controlled, and there was no bruxofacet on splint surface. He discontinued the wear of splint.

\section{DISCUSSION}

Occlusal change has always been a burden to dentist. There are numerous reasons which could cause the occlusal
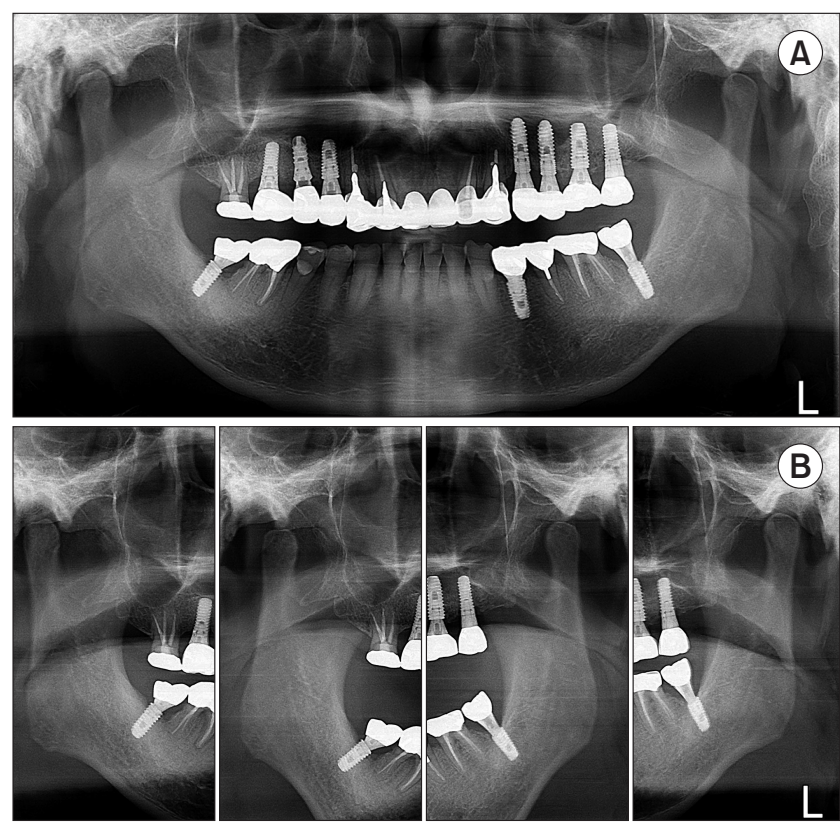

Fig. 6. Case 2. Panorama (A) and temporomandibular joint panorama (B) image taken at the first visit. change except TMD [4-6]. Therefore, dental examination, radiographic inspection, and precise history taking is essential. Various conditions such as pain or effusion of TMJ, degenerative condylar resorption, and articular disc displacement can be a cause of malocclusion [1,2]. Malocclusion could not be a major cause of TMD, but maybe a result of TMD [7]. Occlusion is formed by dentition, TMJ, and masticatory muscle. Based on the results of this study, all teeth had normal contact in the study cast and no pathologic findings were found in the radiographic examination. Therefore, the alteration of occluding teeth must be because of masticatory muscles not because of teeth movement. The cause of alteration of occluding teeth is not for teeth movement, but for masticatory muscles. Without any significant findings in dentition and TMJ, we need to focus on masticatory muscle.

In our study, the first patient did not show any pathological symptom or sign that makes occlusal change. Occlusion is constantly imprinted by tooth contacts with masticatory muscles in the intercuspal position [8]. For this muscular reason, the memory of occlusion can be confused. Then, patients can lose their own occlusion position. A previous study showed the temporary effect of jaw muscle pain on occlusal contact [3]. Splint therapy can help to equilibrate muscular activity of masseter and anterior temporalis [9]. She did not complain of any pain or discomfort, but had nocturnal bruxism. Minor trauma such as clenching or bruxism could be an unexpected cause, and it should be considered in patients with no obvious TMJ symptom [10]. The electromyographic (EMG) amplitude of masseter decreases during sleep, compared to mastication during wakefulness [11]. The size and number of occlusal contacts increase with the activity of masticatory muscle during tooth
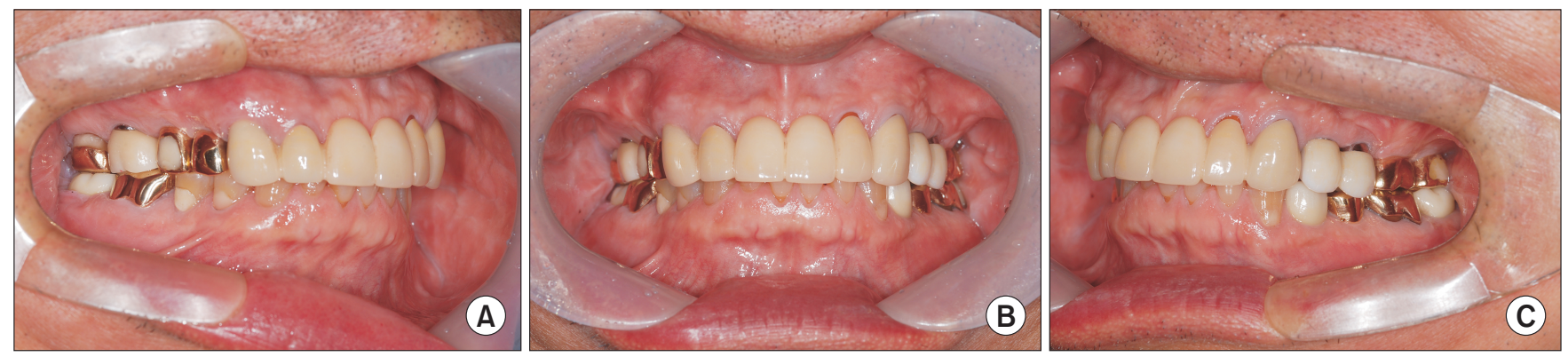

Fig. 7. Case 2. Clinical photographs of the right (A), frontal (B), and left (C) taken after splint treatment. 
clenching [12]. The masseter EMG activity is increased in association with sleep bruxism. Splints can reduce the EMG activity records and improve the balance of masseter muscle activities [13]. Relaxation by splint wearing can help to maintain low EMG activities muscle activity [14]. Occlusal splint has an additional effect on the postural balance [15]. In her case, the stabilization splint was not enough to reduce muscle tension. Botox injections reduce the frequency of bruxism events, decrease bruxism-related disorders [16].

Stabilization splint as a conservative treatment is useful in the management of TMD [17]. Despite its great advantages, several studies had been reported the change of occlusion after inappropriate splint therapy. The mechanism or physiology of possible change after stabilization splint therapy is yet to be fully elucidated. Anterior open bite is more common than posterior open bite. The careless or long-term use of anterior repositioning splint or posterior bite plane usually creates a posterior open bite [18]. In our study, the second patient had been used his splint, but did not visit the clinic during the 2 years of wearing period. On the first visit, he could wear the old splint. The space between teeth and inner surface of splint was irregular, opposite teeth did not contact evenly. Fortunately, all the teeth did not change the position. His occlusal change could be from active lateral pterygoid muscle contraction [19]. Lateral pterygoid muscle play an important role of horizontal jaw movements required in mastication. Although the amounts of jaw shift of the second patient are bigger than the first patient, he got normal occlusion fast. The reason may be that he noticed the change earlier and did not have parafunction. The shorter the patient suffers from the malocclusion, the less time is needed for the symptom resolution.

Nowadays the number of patients who made their splint by self-impression or microwave are growing. Every treatment has pros and cons. This self-fabricated splint has serious side effects that may outweigh their benefit. Patients must receive proper examination and counseling by a dentist before using a splint. After splint delivery, periodic check-ups are also needed.

In conclusion, uncertain occlusal change in patients with or without TMD symptoms was mostly caused by masticatory muscular disorders. Reversible occlusal changes in TMD can be resolved by splint therapy. Therefore, splint therapy is worth considering before occlusal adjustment by tooth grinding, orthodontic, prosthodontic, or surgical treatments.

\section{CONFLICT OF INTEREST}

No potential conflict of interest relevant to this article was reported.

\section{ORCID}

\author{
Ji-Rak Kim \\ https://orcid.org/0000-0002-1326-3948
}

\section{REFERENCES}

1. Marinho LH, McLoughlin PM. Lateral open bite resulting from acute temporomandibular joint effusion. Br J Oral Maxillofac Surg 1994;32:127-128.

2. Chen YJ, Shih TT, Wang JS, Wang HY, Shiau YY. Magnetic resonance images of the temporomandibular joints of patients with acquired open bite. Oral Surg Oral Med Oral Pathol Oral Radiol Endod 2005;99:734-742.

3. Mobilio N, Catapano S. Effect of experimental jaw muscle pain on occlusal contacts. J Oral Rehabil 2011;38:404-409.

4. Craddock HL. Occlusal changes following posterior tooth loss in adults. Part 3. A study of clinical parameters associated with the presence of occlusal interferences following posterior tooth loss. J Prosthodont 2008;17:25-30.

5. Poveda-Roda R, Bagán JV, Sanchis JM, Margaix M. Pseudotumors and tumors of the temporomandibular joint. A review. Med Oral Patol Oral Cir Bucal 2013;18:e392-e402.

6. Elias H, Baur DA. Management of trauma to supporting dental structures. Dent Clin North Am 2009;53:675-689, vi.

7. Manfredini D, Lombardo L, Siciliani G. Temporomandibular disorders and dental occlusion. A systematic review of association studies: end of an era? J Oral Rehabil 2017;44:908-923.

8. Clark JR, Evans RD. Functional occlusion: I. A review. J Orthod 2001;28:76-81.

9. Scopel V, Alves da Costa GS, Urias D. An electromyographic study of masseter and anterior temporalis muscles in extra-articular myogenous TMJ pain patients compared to an asymptomatic and normal population. Cranio 2005;23:194-203.

10. Manfredini D, Peretta R, Guarda-Nardini L, Ferronato G. Predictive value of combined clinically diagnosed bruxism and occlusal features for TMJ pain. Cranio 2010;28:105-113.

11. Kato T, Masuda Y, Yoshida A, Morimoto T. Masseter EMG activity during sleep and sleep bruxism. Arch Ital Biol 2011;149:478-491.

12. MacDonald JW, Hannam AG. Relationship between occlusal contacts and jaw-closing muscle activity during tooth clenching: part I. J Prosthet Dent 1984;52:718-728. 
13. Daif ET. Correlation of splint therapy outcome with the electromyography of masticatory muscles in temporomandibular disorder with myofascial pain. Acta Odontol Scand 2012;70:72-77.

14. Tsai CM, Chou SL, Gale EN, McCall WD Jr. Human masticatory muscle activity and jaw position under experimental stress. J Oral Rehabil 2002;29:44-51.

15. Oliveira SSI, Pannuti CM, Paranhos KS, Tanganeli JPC, Laganá DC, Sesma N, et al. Effect of occlusal splint and therapeutic exercises on postural balance of patients with signs and symptoms of temporomandibular disorder. Clin Exp Dent Res 2019;5:109-115.

16. Shim YJ, Lee HJ, Park KJ, Kim HT, Hong IH, Kim ST. Botulinum toxin therapy for managing sleep bruxism: a randomized and placebo-controlled trial. Toxins (Basel) 2020;12:168.

17. Al-Ani MZ, Davies SJ, Gray RJ, Sloan P, Glenny AM. Stabilisation splint therapy for temporomandibular pain dysfunction syndrome. Cochrane Database Syst Rev 2004;(1):CD002778.

18. Klasser GD, Greene CS. Oral appliances in the management of temporomandibular disorders. Oral Surg Oral Med Oral Pathol Oral Radiol Endod 2009;107:212-223.

19. Murray GM, Phanachet I, Uchida S, Whittle T. The role of the human lateral pterygoid muscle in the control of horizontal jaw movements. J Orofac Pain 2001;15:279-292; discussion 292-305. 